https://helda.helsinki.fi

\title{
Environmental weakening of trophic interactions drives stability in stochastic food webs
}

\section{Ruokolainen, Lasse}

2013

Ruokolainen, L \& McCann , K S 2013 , ' Environmental weakening of trophic interactions pÿdrives stability in stochastic food webs ' , Journal of Theoretical Biology, vol. 339 , pp. 3646 .

http://hdl.handle.net/10138/42974

publishedVersion

Downloaded from Helda, University of Helsinki institutional repository.

This is an electronic reprint of the original article.

This reprint may differ from the original in pagination and typographic detail.

Please cite the original version. 


\title{
Environmental weakening of trophic interactions drives stability in stochastic food webs
}

\author{
Lasse Ruokolainen $^{\mathrm{a}, *}$, Kevin McCann ${ }^{\mathrm{b}}$ \\ ${ }^{a}$ Department of Biosciences, University of Helsinki, Viikinkaari 1, PO Box 65, FI-00014 Helsingin Yliopisto, Finland \\ ${ }^{\mathrm{b}}$ Department of Integrative Biology, University of Guelph, Guelph, Ontario, Canada N1G 2W1
}

\section{H I G H L I G H T S}

- A 3-species food web model is analysed, with stochastic variation in parameters.

- Noise affects either resource growth or consumer intake as a Gaussian function.

- Noise variance, colour, and the consumers foraging adaptation rate is varied.

- Environmental characteristics impact on the consumer-resource interaction strength.

- Noise impact on population variability depends on where it hits in the food web.

\section{A R T I C L E I N F O}

Keywords:

Adaptive foraging

Environmental amplitude

Environmental filtering

Interaction strength

Noise colour

\begin{abstract}
A B S T R A C T
The physiological performance and behaviour of organisms are contingent on environmental conditions. When these conditions vary over time, this can have important consequences for the dynamics of individual populations and entire species communities. While the importance of environmental variation on the structuring and stability of natural food webs is recognised, little work has been done so far to build up a theoretical understanding of these processes. Here we investigate a simple model food web, where a consumer species forages flexibly upon two distinct, inter-competing resource species. Resource productivities or the resource intake rates by the consumer are assumed to depend on environmental conditions in a realistic manner. Fluctuations in these parameters drive variation in the strength of consumer-resource interactions, depending on whether the resource or the consumer is sensitive to environmental noise. The way resources and the consumer respond to changes in environmental conditions stems from the deterministic influence of each stochastic parameter on food web dynamics, which interact with the consumer adaptation process as well as between-resource competition. According to recent empirical reports, the mechanisms behind our results are likely to be relevant in natural systems and thus the model predictions are of potential importance in understanding food web responses to environmental stochasticity in general.
\end{abstract}

(c) 2013 Elsevier Ltd. All rights reserved.

\section{Introduction}

Variation in environmental conditions constitutes an integral part of the dynamics of natural food webs (e.g., Ruokolainen et al., 2009a). Environmental stochasticity affects all aspects of ecosystem functioning, from nutrient cycling (Silvola et al., 1996) to primary production (Ruel and Ayres, 1999), and consumer performance (Englund et al., 2011). Changes in reproduction success, food availability, or foraging ability can be important factors affecting animal behaviour, in terms of migration (Lindén et al., 2011), habitat selection (Abrams, 2000; Abrams et al., 2012, 2007; Amarasekare, 2007), and

\footnotetext{
* Corresponding author. Tel.: + 358919157726.

E-mail address: lasse.ruokolainen@helsinki.fi (L. Ruokolainen).
}

foraging (Abrams and Matsuda, 2004; Abrams, 1999; Post et al., 2000). While the importance of local and spatial heterogeneity for food web dynamics is recognised (Abrams, 2000; Rooney et al., 2008; Ruokolainen et al., 2011), the role of stochastic fluctuations in environmental conditions as a source of such heterogeneity is not yet understood.

In studies of stochastic food webs, environmental variation is assumed to affect either basal resources (Gonzalez and De Feo, 2007; Thébault and Loreau, 2005), intermediate consumers (Vasseur and Fox, 2007), or both resources and their consumers (Ives, 2007; Ripa and Ives, 2003). From these simplified model systems it is known that the system-level consequences of environmental forcing depend on which nodes in the food web are being affected by external perturbations (Greenman and Benton, 2005a). The impact of environmental variation can also depend on the strength of species 
interactions within the food web (Ripa and Ives, 2003; Ruokolainen and Ripa, 2012; Ruokolainen et al., 2009b) and temporal characteristics of environmental fluctuations, such as variance and colour (Ruokolainen et al., 2009a). Variation in the colour (referring to the dominant mode of fluctuations; blue for fast and red for slow) of environmental fluctuations can have a different effect on species embedded in food webs, depending on their trophic position (affecting their demographic characteristics) (Greenman and Benton, 2005a, 2005b). A general pattern in simple systems is that species with slow (fast) dynamics are destabilised (stabilised) by increasing environmental reddening (Ruokolainen et al., 2009a). Moreover, both environmental amplitude (variance) (Vasseur and Fox, 2007) and colour (Ripa and Ives, 2003) can interact with consumer-resource cycles, affecting food web stability.

Adaptive behaviour is considered to be important for shaping local (Abrams and Matsuda, 2004; Abrams, 2000; Ma et al., 2003; Post et al., 2000; Teng and McCann, 2004) and regional (Abrams and Ruokolainen, 2011; Abrams et al., 2012; Amarasekare, 2008, 2007; Ruokolainen et al., 2011) population dynamics, food web structure (e.g., Beckerman et al., 2006; Heckmann et al., 2012), and species persistence (Kondoh, 2006, 2003; Uchida and Drossel, 2007). Dynamic coupling of distinct food chains by an actively foraging top predator is regarded as important for community stability (e.g., Abrams, 2000; McCann et al., 2005; Rooney et al., 2008; Teng and McCann, 2004). This means that mobile predators can integrate over disparate energy sources (Vander Zanden and Vadeboncoeur, 2002), and pursue relatively more abundant prey while decoupling from scarce prey (McCann and Rooney, 2009). While such behaviour can destabilise the dynamics of individual food chains, asynchronous dynamics between them ensures that dynamics of the entire food web become more stable.

Abiotic perturbations and behavioural dynamics are both important drivers of population fluctuations in food webs. However, at present it is not clear how behavioural dynamics arising from trophic coupling might interact with environmental fluctuations, and how this in turn affects the stability of food webs. Here we study simplified food webs, consisting of two resources and one consumer, foraging adaptively upon the resources, which are assumed to compete with each other, in resemblance of so-called diamond-shaped food webs studied elsewhere (e.g., McCann and Rooney, 2009). The system is affected by environmental fluctuations, such that the vital rates of either resources or the consumer vary along these fluctuations in a realistic manner. Both biological parameters are assumed to have a unimodal response to varying environmental conditions, reflecting patterns in natural systems (e.g., Englund et al., 2011). We ask how characteristics of environmental stochasticity (variance and colour), and the point of environmental impact (either resources or the consumer) affect food web dynamics under adaptive consumer foraging. The consumer changes its preference on the two resources flexibly, depending on their relative profitability.

In what follows we will first introduce the deterministic skeleton of our food web model and describe the relationships between the parameters of this model with environmental stochasticity. Next we will outline characteristic patterns in the distribution of the stochastic parameters under varying environmental properties, such as variance and temporal colour. Here we will show that these patterns underlie characteristic effects of environmental fluctuations on population stability, due to variation in the interaction strength between the resources and the consumer, arising from (i) variation in mean parameter values, and (ii) the way populations react to periods of low resource growth or resource consumption. Such periods promote consumer decoupling from resources, which has important consequences for food web dynamics. This suggests that a consumer's adaptation potential can interact with stochastically driven variation in resource productivity or consumer intake rates.

\section{Methods}

\subsection{Food web model}

Population dynamics in a diamond-shaped food web (McCann et al., 1998; McCann and Rooney, 2009; Vasseur and Fox, 2007) are given by the following differential equations:

$$
\frac{d N_{i}}{d t}=N_{i}\left[r_{i}-k_{i}\left(N_{i}+b N_{j}\right)\right]-\frac{\alpha_{i} I_{i} N_{i} P}{\sum_{j} \alpha_{j} N_{j}+B_{0}}
$$

$\frac{d P}{d t}=P\left(\frac{e \sum_{i} \alpha_{i} I_{i} N_{i}}{\sum_{j} \alpha_{j} N_{j}+B_{0}}-d\right)$

$\frac{d \alpha_{i}}{d t}=g \alpha_{i} e\left(\frac{I_{i} N_{i}}{\sum_{k} \alpha_{k} N_{k}+B_{0}}-\frac{\sum_{j} \alpha_{j} I_{j} N_{j}}{\sum_{k} \alpha_{k} N_{k}+B_{0}}\right)$,

where Eq. (1.a) specifies the dynamics of two resources $N_{i}$, growing logistically in the absence of consumption. The parameterisation of the logistic used here separates the per capita resource growth rate into a maximum per capita growth rate $r$ and a per capita reduction in that rate of $k$, giving a carrying capacity of $r / k$ (Abrams and Ruokolainen, 2011; Rueffler et al., 2006). The resource populations compete for a shared explicit resource. The intensity of this inter-specific competition relative to the strength of intra-specific competition is defined by $b$. Resource populations are foraged by a consumer species $P$, with a saturating functional response. Resource consumption depends on the consumer preference on each resource $\alpha_{i}$ (proportion of resource $i$ in the consumers diet), the maximum intake rate $I_{i}$, and the halfsaturation constant $B_{0}$. The consumer (Eq. (1.b)) converts ingested resource to its own biomass with efficiency $e$. Consumer growth is reduced by a constant loss rate $d$.

Consumer preference for resource $i$ changes adaptively through time according to Eq. (1.c), depending on adaptation rate $g$ (Kondoh, 2003; 2006; Matsuda et al., 1996). This so-called 'replicator equation' weights the fitness of a particular behaviour against the average fitness. Originally, this equation was introduced to describe evolution in a population consisting of $n$ different types. The replicator equation then models the changes in the frequency of each type, depending on their fitness in comparison with the average fitness within the population (Hofbauer and Sigmund, 1998). Here foraging on resource $i$ is increased (decreased), if the potential energy gain from consuming that resource alone is greater (lower) than the expected energy gain from feeding on both resources. This constrains the consumer preferences $\alpha_{i}$, such that $\Sigma \alpha_{i}=1$.

Following earlier work on adaptive consumer foraging, parameter values are chosen to generate cyclic population dynamics in the absence of consumer adaptation and environmental stochasticity (e.g., Abrams and Ruokolainen, 2011; Rooney et al., 2006; Vasseur and Fox, 2007). We set $r_{i}=0.65, k_{i}=1, b=0.25, I_{i}=0.65$, $B_{0}=0.15, e=0.5$, and $d=0.1$. In the absence of stochasticity, and assuming random consumer foraging, increasing either $r_{i}$ or $I_{i}$ is associated with a Hopf bifurcation. This happens at $r \approx 0.354$ and $I \approx 0.362$, respectively (see Fig. S1 in Supplementary material); the exact point of the bifurcation can only be determined numerically. When simulating the system under stochastic environmental variation, consumer adaptation is assumed to be $g=0.5$, which is an intermediate rate between very slow $(g=0.01)$ and very fast $(g=10)$. The robustness of our results against variation in the model parameters, as well as the underlying stability of the community equilibrium, were evaluated by analysing 1000 randomly drawn parameter sets (randomising $b, B_{0}, e$, and $d$, while keeping $r, k$, and $I$ constant), with all species persisting at the equilibrium (equilibrium density $\geq 0.1$ ), assuming either cyclic or stable equilibrium dynamics. 
A

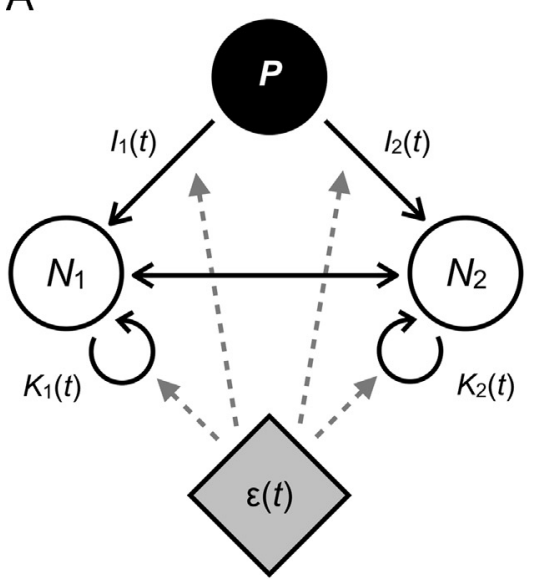

B

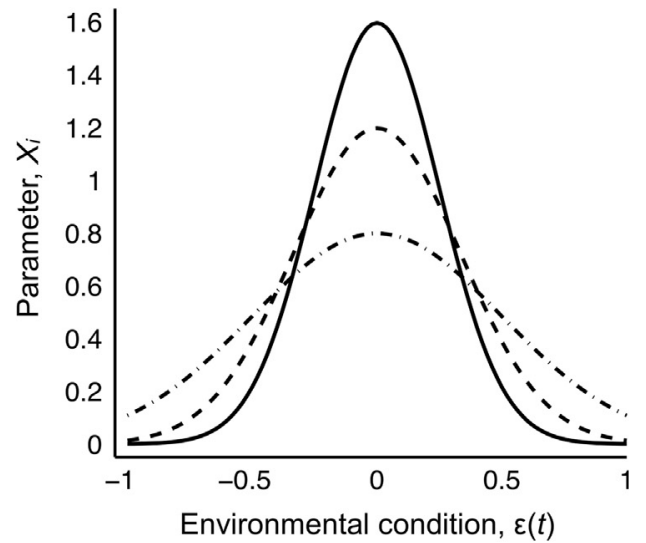

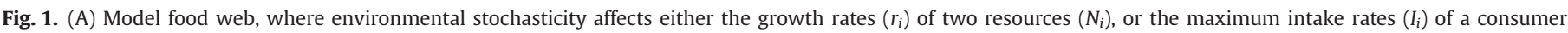

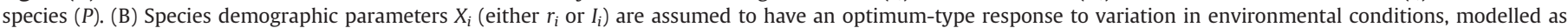

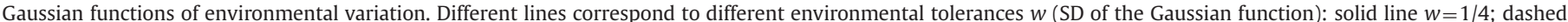
line $w=1 / 3$; dash-dotted line $w=1 / 2$.

\subsection{Environmental stochasticity}

We are here interested in the effects of environmental forcing on food web dynamics. More specifically, we investigate how environmental variation that directly affects either resources or the consumer influences different aspects of individual populations and the entire food web. Previously, noise has been introduced into the population per capita growth rates in discrete-time (Greenman and Benton, 2005b) and continuous-time (Xu and Li, 2003) food chain models to demonstrate that the 'point of entry' of noise in the food chain can affect the colour (spectral density) of population dynamics. This approach is, however, somewhat problematic when considering noisy consumer dynamics; population growth can be positive even in the absence of food under favourable environmental conditions. For simple consumer-resource models a mechanistic approach for generating temperature-dependent population dynamics has been proposed, based on metabolic theory (Vasseur and McCann, 2005; Vasseur, 2007). While this methodology is highly attractive, its complexity hampers the tractability of environmental perturbations in more complex food webs. Therefore we utilise a somewhat simpler approach for introducing noise into our food web model.

When resources are sensitive to noise, we assume that resource growth rate $\left(r_{i}\right)$ varies over time. For consumer species it is realistic to assume that environmental fluctuations affect consumptive parameters, such as the maximum intake rates $I_{i}$ (Englund et al., 2011) (Fig. 1A). Stochastic parameters are assumed to have an optimum-type response to noise (Englund et al., 2011; Laakso et al., 2001), here modelled as a Gaussian function of environmental variations

$X_{i}(t)=\frac{1}{\sqrt{2 w \pi}} \exp \left[-\frac{\left(\mu_{i}-\varepsilon_{i}(t)\right)^{2}}{2 w^{2}}\right]$,

where $X$ is the stochastic parameter of interest $\left(r_{i}\right.$ or $\left.I_{i}\right), \varepsilon_{i}$ is species-specific environmental noise, $\mu_{i}$ is the species environmental optimum, and $w$ is the species environmental tolerance (Fig. 1B). For simplicity, we assume that all species have their optima at the long-tem environmental mean (i.e., $\mu_{i}=0$ ). In the following analyses we assume that $w=1 / 3$, resulting in $E\left[X_{i}(t)\right] \approx$ 0.65 , when environmental amplitude is set to $\sigma=0.5$.

Environmental noise $\varepsilon_{i}$ is modelled as a $1 / f$ process (Halley, 1996; Ruokolainen, et al., 2009a; Vasseur and Yodzis, 2004), by summing up $T / 2$ sine waves with random phases, where $T$ is the length of the noise time series

$\varepsilon_{i}(t)=\sum_{f=1}^{T / 2} \frac{1}{f^{\gamma / 2}} \sin \left(\frac{2 \pi f t}{T}+\theta_{i}(f)\right)$,

where $t$ is time, $f$ is frequency, $\gamma$ is the spectral exponent, and $\theta_{i}(f)$ is a vector of random phases, independently drawn from a uniform distribution between $[0,2 \pi]$. Parameter $\gamma$ determines the 'colour' the noise time series (Vasseur and Yodzis, 2004), indicating the rate of change in environmental conditions over time. When $\gamma=0$ noise is said to be white (all frequencies have equal weight) and there is no serial dependency between observations. Setting $\gamma>0$ leads to an increasing dominance of low frequencies in the time series seen as slow variation and strong serial dependency between observations. This range of $\gamma$ is collectively referred to as red noise. Special cases include 'pink' noise $(\gamma=1)$, 'brown' noise $(\gamma=2)$, and 'black' noise $(\gamma \geq 3)$. Pink noise has been proposed as a null model for environmental variation (Vasseur and Yodzis, 2004). Here we will consider an ecologically realistic range of noise colours $0 \leq \gamma \leq 2$ (Vasseur and Yodzis, 2004). Upon generation, noise time series are standardised and scaled to a desired asymptotic variance $\sigma^{2}$. Finally, we assume that noise affecting different components of the food web model are independent.

The filtering of environmental fluctuations (Fig. 1B) affects species demographic parameters, depending on the amplitude of $\varepsilon_{i}$. As the maximum performance is attained at the optimum, increasing deviations from this optimum lead to a reduction in the longterm mean of stochastic parameters (Fig. 2A). While the average parameter values always remain in the region associated with cyclic population dynamics in a deterministic system (Fig. S1), stochastic variation at each time point can bring the system to a region of stable equilibrium dynamics (Fig. 2B). However, increasing noise amplitude also increases the risk of $r_{i}$ or $I_{i}$ becoming too low to support consumer growth (Fig. 2B). Environmental reddening (increasing $\gamma$ ) is known to increase the occurrence of runs of extreme conditions (Fowler and Ruokolainen, 2013a; Schwager et al., 2006). In the present case environmental reddening increases the expected length of periods associated with stable equilibrium dynamics (and periods of unfavourable conditions for the consumer) (Fig. 2C). These aspects of environmental stochasticity outlined in Fig. 2 have important consequences for food web dynamics. 
A

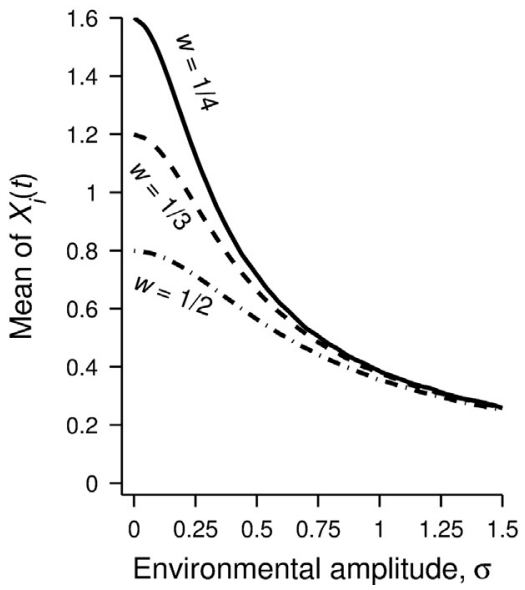

B

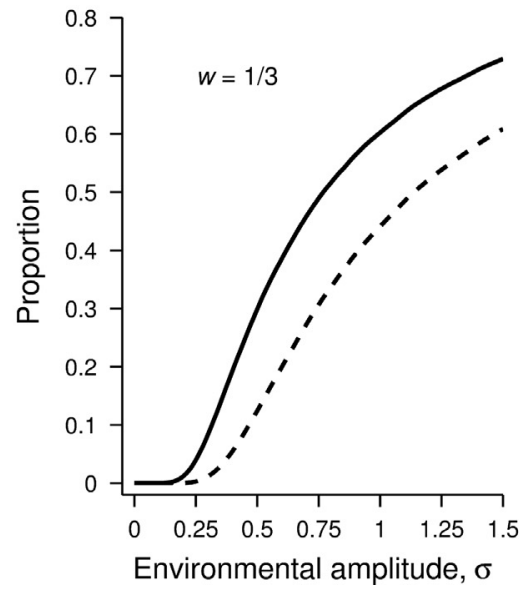

C

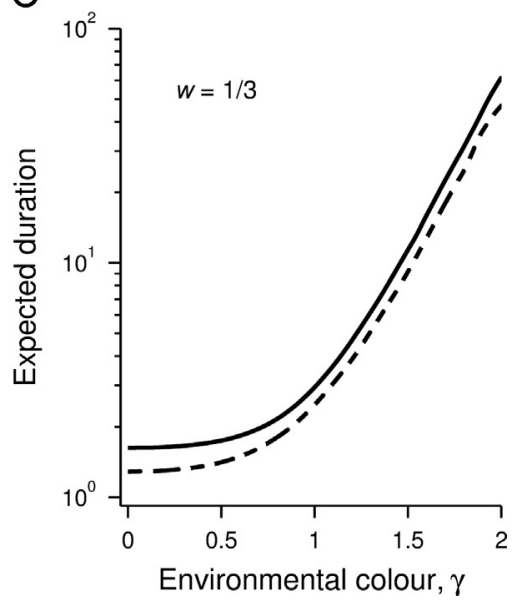

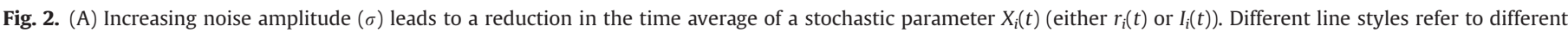

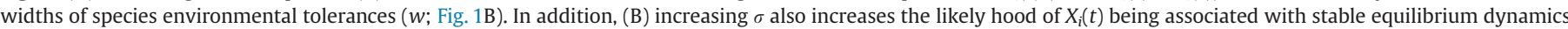

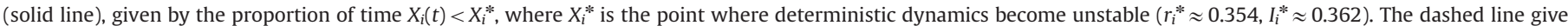

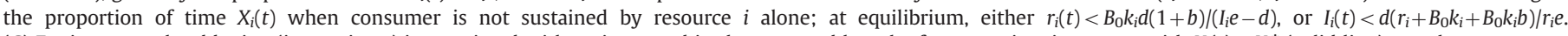

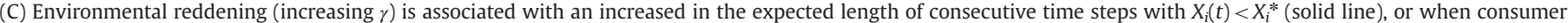
is not sustained by resource $i$ alone (dashed line). In (A, B) $\gamma=1$, and in (C) $\sigma=0.5$.

\subsection{Analysis}

The scope of this paper is to analyse how different aspects of environmental stochasticity affect dynamics at the levels of the entire food web and individual populations, when either the resources or the consumer is sensitive to environmental fluctuations. Environmental characteristics that are known to affect food web dynamics include the temporal mean (Vasseur and McCann, 2005), fluctuation amplitude (Gouhier et al., 2010; Vasseur and Fox, 2007), spectral colour (Gonzalez and De Feo, 2007; Greenman and Benton, 2005a), and the similarity of species environmental responses (Ripa and Ives, 2003; Ruokolainen and Ripa, 2012; Thébault and Loreau, 2005). Here we concentrate on analysing the effects of varying environmental amplitude $\left(\sigma^{2}\right)$ and colour $(\gamma)$. We also consider the effect of varying consumer adaptation rate $(g)$, as different speeds of behavioural dynamics can potentially resonate with the consumer-resource cycle in different ways, depending on the regime of environmental variation.

We simulate the food webs numerically using the second-order Adams-Bashforth method (Butcher, 2008), with a step size $d t=0.1$ time units (environmental variation is updated for each full time unit). The system is simulated for $T=10000$ time units, and the first 5000 are discarded prior to analysis. The remaining time series are used to calculate the coefficient of variation $(C V=\mathrm{SD} /$ mean $)$ of total food web biomass and that of individual species (resource or consumer). We also calculate the cross-correlation between resource populations (resource synchrony), which is considered as a good proxy for stability (Abrams and Ruokolainen, 2011; e.g., Teng and McCann, 2004).

\section{Results}

\subsection{Environmental characteristics}

When noise impacts on resource growth rates $\left(r_{i}\right)$, the effect increasing noise amplitude $(\sigma)$ has on the size of food web biomass fluctuations depends on environmental colour $(\gamma)$. In general, the reduction in the mean $r_{i}$ associated with increasing $\sigma$ (Fig. 2A) has a stabilizing effect on the consumer-resource interactions (Fig. 2B; Rip and McCann, 2011), explaining why the $C V$ of food web biomass decreases with increasing $\sigma$ under white environments (Fig. 3A). This effect is relatively strong, as biomass $C V$ decreases even though both resources and the consumer decline in density (Fig. 4A, C). Environmental reddening (increasing $\gamma$ ) leads to increasing biomass $C V$ under intermediate to strong $\sigma$ (Fig. 3A). Under weak forcing food web dynamics always remain cyclic (Fig. 2B). Reduced mean growth rates at higher environmental amplitudes make the resources more responsive to environmental reddening, leading to slow, asynchronous fluctuations between resources, which are further amplified by competition. This results in increasingly long time periods when one of the resources is at very low density (both due to low $r_{i}(t)$ and competition). Such periods favour the consumer to concentrate more on the resource with the higher productivity $\left(r_{i}(t)\right.$ and $\left.K_{i}(t)=r_{i}(t) / k\right)$, which is prone to amplify the $N-P$ cycles. Together, these two factors explain why environmental reddening increases population and biomass $C V$ under relatively high noise amplitudes (Fig. 3A, C, E).

In contrast, when noise affects consumer intake rates $\left(I_{i}\right)$, increasing noise amplitude stabilises food web biomass variability independently of noise colour (Fig. 3B). This is paralleled by decreased resource variability (Fig. 3D), which is in turn associated with an increase in resource density. These patterns are due to reduced predator intake rates, having a stabilising effect on the $N-P$ interactions (Fig. S1; Rip and McCann, 2011). The increase in resource densities (due to reduced predation pressure) initially promotes consumer density (Fig. 4D). However, increasing either environmental amplitude $(\sigma)$ or colour $(\gamma)$ increases the likelihood that the consumer is temporally supported by only one (or even none) of the resources (Fig. 2B, C)-intake rates being too low to compensate for the loss rate $(d)$. This lets resources escape consumption under periods of sufficiently low $I_{i}(t)$, which dampens resource fluctuations as less stochasticity is transmitted from the consumer. Competition from the non-preferred resource further supresses the density on the preferred resource, leaving the consumer with less food available. Patterns in consumer variability (Fig. 3F) then reflect those in consumer mean density (Fig. 4D). Increasing either $\sigma$ or $\gamma$ both increase the likelihood and duration of periods of starvation, explaining the decline in consumer density.

\subsection{Consumer adaptation rate}

As in the case of environmental characteristics, how population and food web level variability respond to varying consumer adaptation rate $(g)$ can depend on which parts of the system are sensitive to noise. In general, the stability of food web dynamics 
A

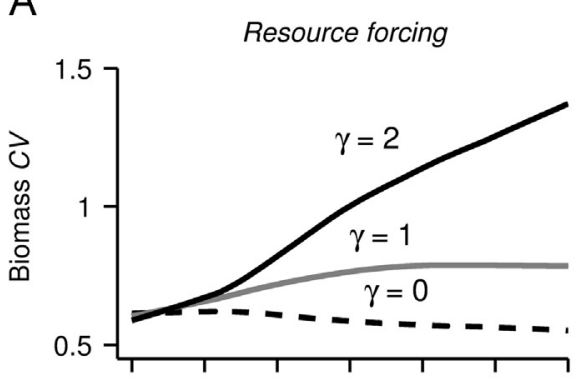

C

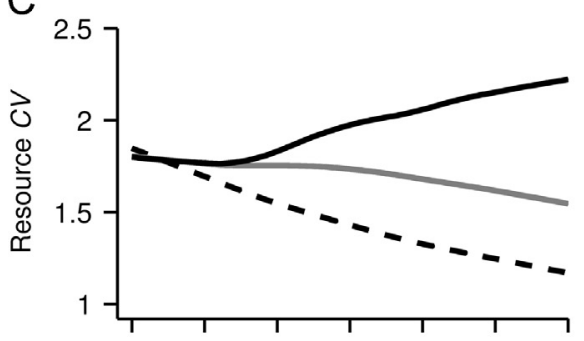

E

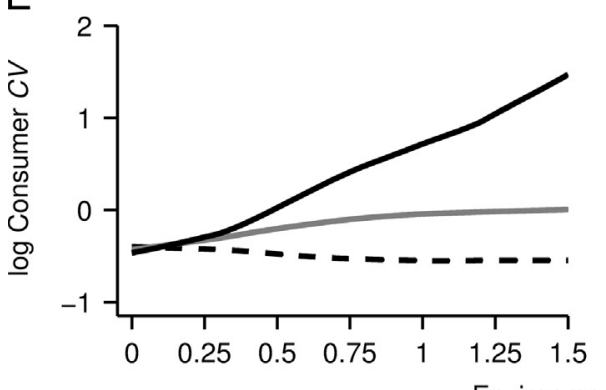

B

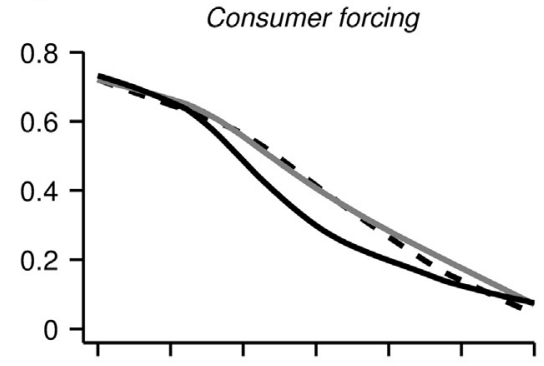

$\mathrm{D}$

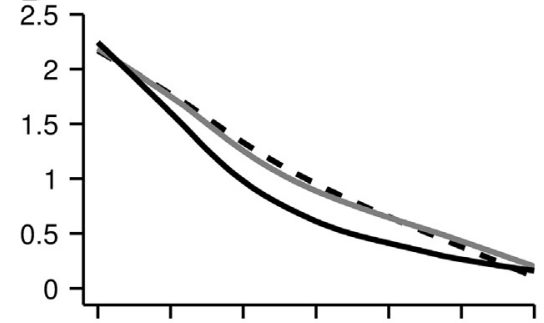

$\mathrm{F}$

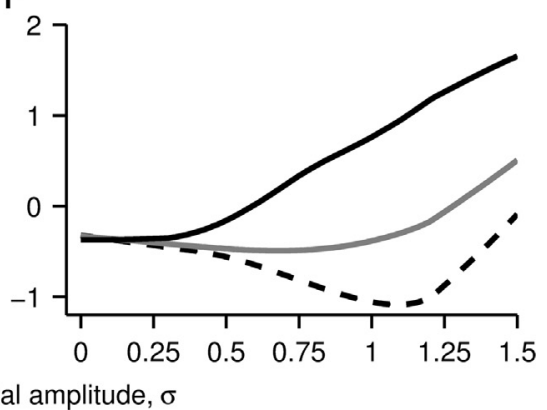

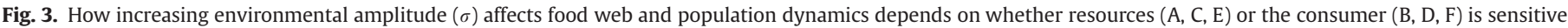

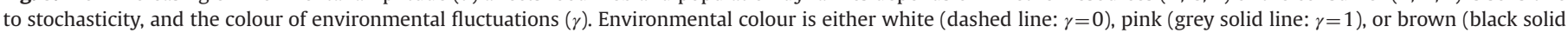

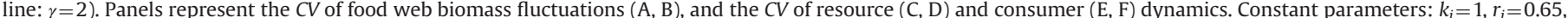
$I_{i}=0.65, B_{0}=0.15, e=0.5, d=0.1$, and $g=0.5$. (For interpretation of the references to color in this figure legend, the reader is referred to the web version of this article).

affects the system's sensitivity to adaptive foraging (Post et al., 2000). As environmental amplitude affects the average values of stochastic parameters (Fig. 2A), and as varying either $r_{i}$ or $I_{i}$ has a different effect on underlying population dynamics (Fig. S1), it is not surprising that population responses are more sensitive to varying $g$ when noise affects resource growth rates than when it affects consumer consumption rates (Fig. 5A-D).

Under relatively low noise amplitudes, increasing $g$ tends to destabilise resource fluctuations (Fig. 5A, B) and stabilise consumer fluctuations (Fig. 5C, D). This pattern arises due to the consumer switching between the two resources, which on one hand amplifies resource dynamics, but on the other hand promotes asynchronous resource fluctuations (Fig. 5E, F). Asynchrony in the consumers resource base leads to the so-called 'bird feeder effect', where the consumer encounters a relatively stable food supply by constantly switching between resources (McCann and Rooney, 2009).

Increasing noise amplitude quickly reduces the sensitivity to varying $g$, such that at $\sigma=0.5$ increasing the consumer adaptation rate has almost no influence on the long-term patterns in population dynamics. This arises namely due to the increased prevalence of stable equilibrium dynamics (Fig. 2B), increasing the relative contribution of stochastic variation in population fluctuations. However, qualitative differences appear in population time series between low and high adaptation rates, as well as between scenarios of the point of environmental impact. Under stochastic $r_{i}$ 's slow adaptation is associated with long time-periods with only one resource available to the consumer (Fig. 6A); the consumer does not abandon the resource with low $r_{i}(t)$, leading to a collapse of this resource. With fast adaptation the consumer usually uncouples from a declining resource (unless the other resources has even lower energetic value due to a low $\left.r_{i}(t)\right)$, which acts to prevent such collapses. In contrast to the previous case, stochastic $I_{i}$ 'S and slow adaptation are associated with a relatively high resource synchrony (under random foraging resource are fully synchronised in the absence of noise) without resource collapses (Fig. 6B). Again, fast adaptation asynchronous short-term fluctuations in resource densities. This beneficial effect of fast adaptation requires that the environmental variables $\varepsilon_{i}(t)$ are not strongly correlated, as this makes fitness differences in engaging different prey to diminish.

\section{Discussion}

The sensitivity of different species in food webs to environmental variation can be important for population dynamics: responses to changes in environmental characteristics-such as variance and temporal colour, or adaptive consumer behaviour can differ qualitatively, depending on whether environmental fluctuations affect resource productivities $\left(r_{i}\right)$ or consumer intake rates $\left(I_{i}\right)$. The influence of stochasticity on food web dynamics in our model 
A

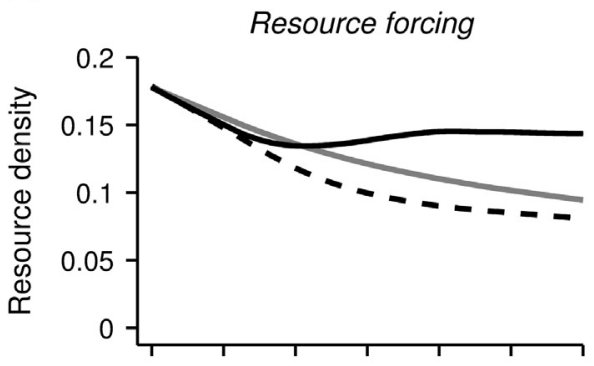

C

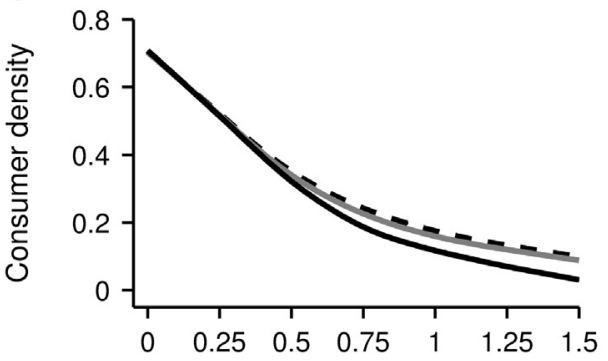

B

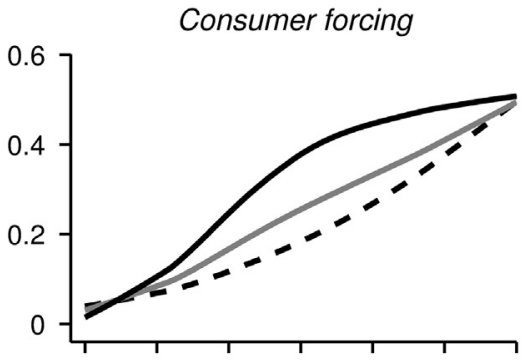

$\mathrm{D}$

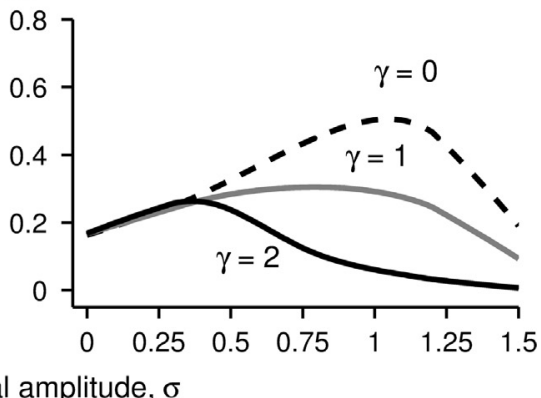

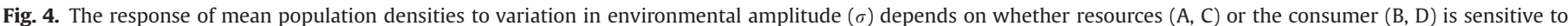

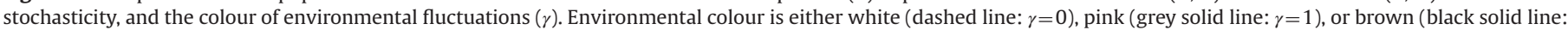

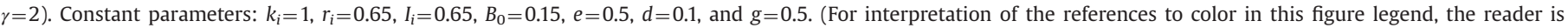
referred to the web version of this article).

A

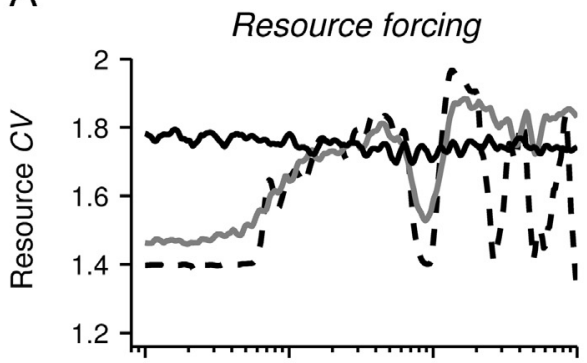

C

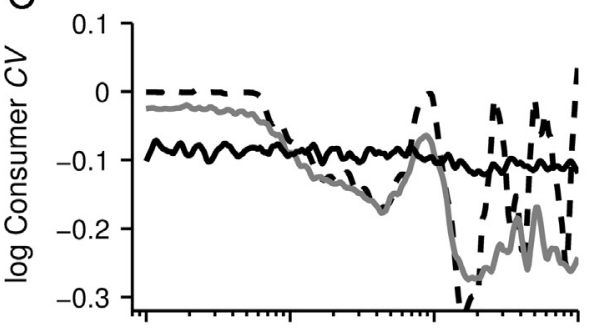

$\mathrm{E}$

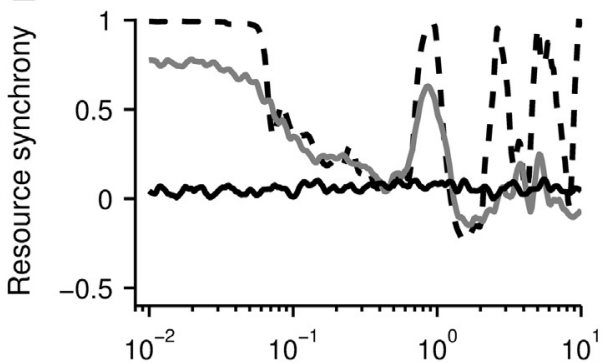

B

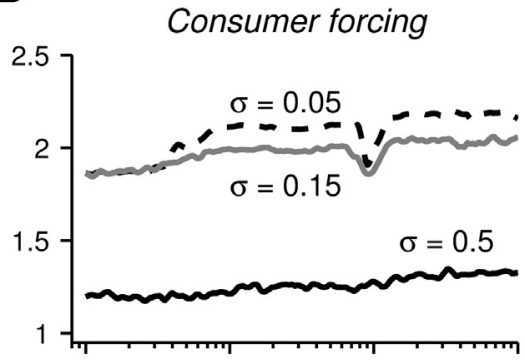

$\mathrm{D}$

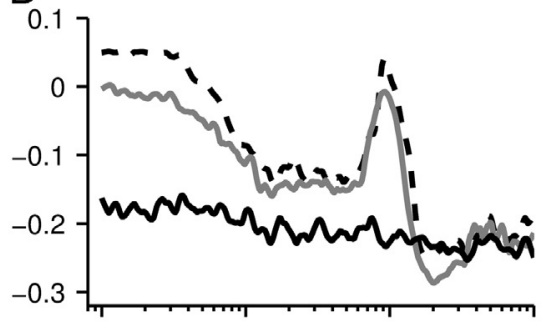

$\mathrm{F}$

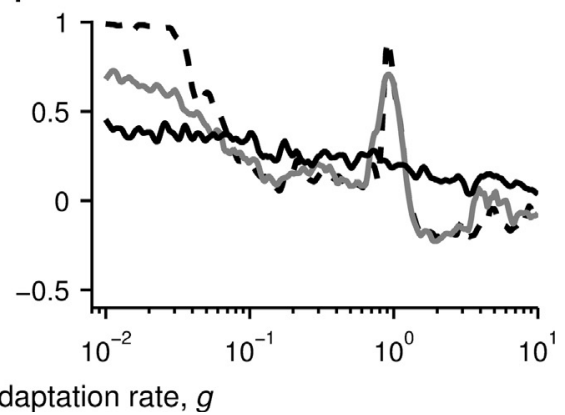

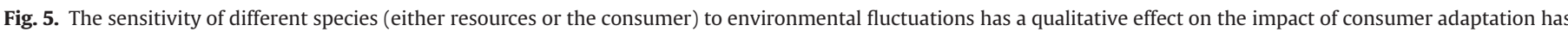

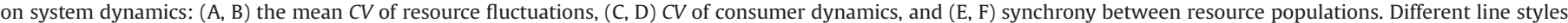
refer to the amplitude of environmental noise $(\sigma)$. Constant parameters: $k_{i}=1, r_{i}=0.65, I_{i}=0.65, B_{0}=0.15, e=0.5, d=0.1$, and $\gamma=1$. 
A
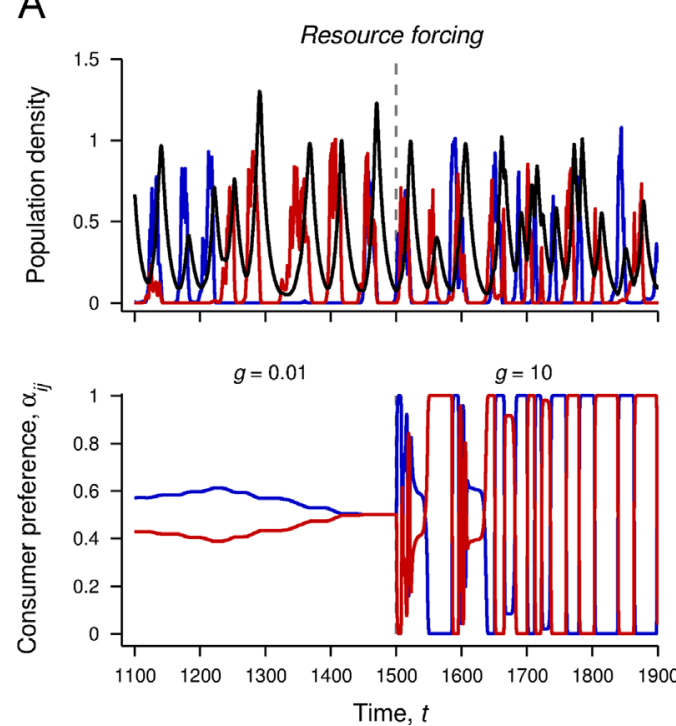

B
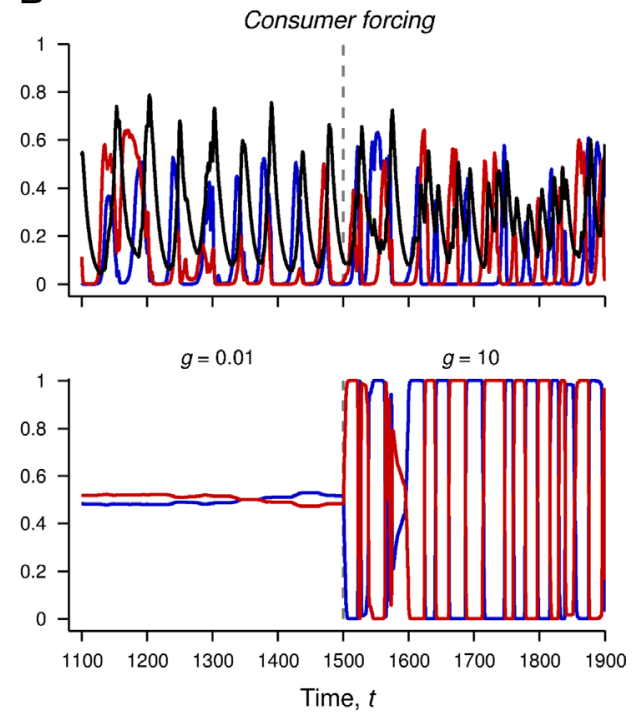

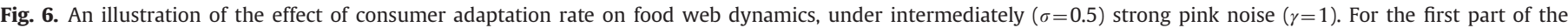

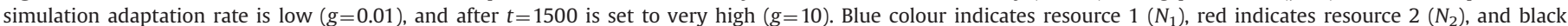

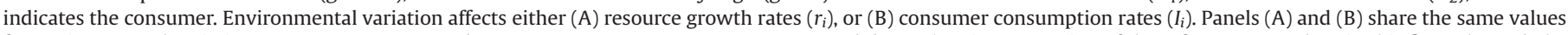

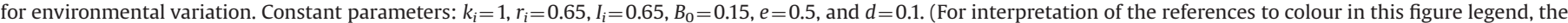
reader is referred to the web version of this article.)

arises due to an interaction between environmental fluctuations and the optimum-type response of biological rates to these fluctuations (a filtering process). Many results can be understood via patterns in the occurrence of periods of relatively low resource productivity or consumption (Fig. 2).

Increasing noise amplitude reduces the average of the stochastic parameter $\left(r_{i}\right.$ or $\left.I_{i}\right)$, which in turn weakens the consumer-resource interactions and stabilises population fluctuations. Stronger noise increases the likelihood of the underlying dynamics becoming stable, but it also leads to situations where the consumer is temporally unsupported by one (or both) of the resources. Environmental reddening increases the duration of such conditions. While this promotes consumer decoupling from resources, the consequences for food web dynamics are quite different, depending on whether when noise affects $r_{i}^{\prime}$ s or $I_{i}^{\prime}$ s. Under fluctuating resource productivities the consumer prefers to forage on the resource with the higher $r_{i}(t)$, which actually destabilises temporal dynamics. When consumption rates vary stochastically, low $I_{i}(t)$ lets a resource to escape consumption, which has a stabilising effect on long-term dynamics.

When considering the interaction between environmental variation and consumer adaptation, the effect of noise can again depend on whether $r_{i}$ or $I_{i}$ are sensitive to stochasticity. The adaptation process is sensitive to the stability of consumerresource interactions, and variation in environmental amplitude has different effects on the size of population fluctuations depending on which parameters are sensitive to stochasticity (Fig. S1). While the influence of adaptive consumer behaviour on food web dynamics can differ between scenarios of environmental impact under weak noise or when adaptation is slow, fast adaptation promotes asynchronous resource fluctuations under higher environmental amplitudes independently of the targeting of noise.

\subsection{The point of environmental impact and why this matters}

When subjected to environmental noise, the colour (spectrum) of population fluctuations becomes red-shifted for species higher up in a food chain (Greenman and Benton, 2005a; Ripa et al., 1998); higher-order consumers have slower dynamics than basal resources, which translates to amplification of low-frequency fluctuations in the environmental noise (Kaitala et al., 1997; Ripa and Heino, 1999). Given that trophic links are biased (which they usually are), the direction in which environmental perturbations are transmitted through a food web has an effect on population spectra (Greenman and Benton, 2005a). This means that both population colour and variance can depend on which population(s) in a food web is influenced by stochasticity.

Here we do not simply introduce environmental variation as additive noise to population per capita growth rates (as, e.g., in Greenman and Benton, 2005b; Ripa and Ives, 2003), but instead let specific parameters to depend on environmental fluctuations in a realistic manner (Englund et al., 2011; e.g., Thébault and Loreau, 2005; Vasseur and McCann, 2005). These approaches generate different predictions regarding increasing environmental amplitude-while the traditional method predicts increasing population variances, population fluctuations can actually be dampened in our model. This happens because increasing environmental variance reduces the mean of stochastic parameters (having unimodal response to environmental variation), leading to weakened consumer-resource interactions (e.g., McCann et al., 1998; Rip and McCann, 2011).

Vasseur and Fox (2007) studied a diamond-shaped food web, where the death rates of two intermediate consumers were multiplicatively affected by stochasticity. They reported that an initial increase in noise amplitude stabilised consumer population dynamics, while a further increase was destabilising (Vasseur and Fox, 2007). This finding resembles our result when consumer intake rates vary under white noise (Fig. 3F). In their model increasing environmental amplitude increases the mean consumer loss rate, which in turn decreases the net energy flux from the resource to the consumers, having a stabilising effect on population dynamics (Rip and McCann, 2011). However, increasing environmental amplitude increases the likelihood that consumer loss rate exceeds the amount of energy gained from a resource $\left(e I_{i} R_{i} /\left(R_{i}+R_{0}\right)<d\right)$, which in our model leads to the consumer tending to become uncoupled from such a resource. This initially reduces both resource and consumer variability, but eventually leads to increasing consumer starvation. 
In general, differences between model predictions are likely to arise due to three factors. Firstly, the nature of deterministic population dynamics can have an influence on how perturbations are filtered through the food web; environmental weakening of interactions associated with stable dynamics can in some cases have a different result to that of unstable dynamics (Fig. S2). Secondly, food web composition is likely to play an important role in how environmental fluctuations modify food web dynamics (Greenman and Benton, 2005b). That is, a response of a population to a perturbation is contingent on the patterns in between-species interactions in the food web, as well as on the identity of species targeted by the perturbation. Thirdly, in addition to the trophic location of environmental impact, which parameter(s) is affected by noise can also have a qualitative effect on how environmental variation affects population stability. However, these factors are unlikely to affect our main conclusions that the impact of environmental variation can be understood by considering the deterministic roles of stochastic parameters as well as their temporal distribution.

When environmental variation affects different aspects of population growth (affecting either resources or the consumer), it is not surprising that the underlying mechanism driving population stability differs between scenarios. Introducing noise into model parameters affects patterns in dynamical stability (Vasseur and McCann, 2005), and the effect will vary when different parameters are considered. When resource growth rates $\left(r_{i}\right)$ vary stochastically, reduced mean resource carrying capacity $(K=r / k)$ leads to a decline in population densities, but is also associated with higher dynamical stability (Rip and McCann, 2011). In contrast, under stochastic consumer intake rates $\left(I_{i}\right)$ reduced mean resource intake promotes higher resource densities. This increase in food availability can in turn increase consumer densities, until intake becomes too low for consumers to effectively utilise abundant resources. The same effect can arise when increasing consumer mortality, e.g., due to harvesting (Abrams, 2009; Abrams et al., 2012). These results demonstrate that the way food webs are affected by environmental fluctuations can have important, and previously unrecognised dynamical consequences.

\subsection{Environmental colour}

Vasseur (2007) analysed a simple consumer-resource model, where ambient temperature was mechanistically related to species biological rates. He showed that the stability of resource and consumer population fluctuations react differently to environmental reddening; resource dynamics are more stable in strongly reddened environments than in white environments, while consumer fluctuations are destabilised by reddening. These results were related to two factors: (i) the resource (consumer) displayed overcompensatory (undercompensatory) dynamics, which leads to amplification of reddened environmental noise (Petchey et al., 1997; Ripa and Heino, 1999); (ii) environmental noise had a stronger (weaker) effect on the target densities (temporal equilibria) of the consumer (resource), again increasing (decreased) population variance in red environments (Greenman and Benton, 2005a). Using a linear model with one consumer feeding upon two resources, Ripa and Ives (2003) demonstrated that the period of the consumer-resource cycle resonates with environmental noise, such that population variances are usually maximised at some intermediate environmental autocorrelation between white and red noise. The period length of the consumer-resource cycle is influenced by the strength of interactions between the consumer and the resources, which in turn determines the location of the variance maximum along varying environmental colour (Ripa and Ives, 2003). Ruokolainen and Ripa (2012) suggested that the interaction strength could shift the consumer and resource variance maxima with respect to each other, which could explain why Vasseur and McCann (2005) observed maximum variability at an intermediate noise colour only for the resource population.

In our model environmental amplitude and temporal colour interact in affecting the likelihood and expected duration of conditions associated with stable equilibrium dynamics, or the consumer being unsupported by one of the resources (Fig. 2B, C). Such conditions do not arise unless noise amplitude is sufficiently high, which explains why environmental reddening does not affect population dynamics under weak noise. Further, how environmental reddening affects population dynamics depends on whether noise affects resource productivity $\left(r_{i}\right)$ or consumer resource intake $\left(I_{i}\right)$. Uncorrelated (between-species), reddened environmental variation amplifies fluctuations in the densities of competitors (Ruokolainen and Fowler, 2008; Ruokolainen and Ripa, 2012). In addition to the fact that the consumer couples more strongly to the resource that is favoured by the environment (having a relatively high growth rate and carrying capacity, which excites the consumer-resource cycle; Rip and McCann, 2011), this explains why environmental reddening destabilises food web dynamics under stochastic $r_{i}^{\prime}$ s. Stochastically reduced $I_{i}^{\prime}$ $\mathrm{s}$ also lead to the consumer independently uncoupling from the resources. This lets the resources to recover towards their deterministic carrying capacities, increasing their long-term densities. Further such a resource not favoured by the consumer competitively suppresses the other resource, which reduces consumer density.

Gonzalez and De Feo, (2007) studied a system where several consumers competed over a common resource, with consumer intake rates being affected by environmental stochasticity, in a somewhat similar way as done here. In their model consumers had different intake optima along an environmental gradient which affected their relative performance under varying conditions. They reported increased consumer population variability in association with environmental reddening, which matches our results. In their model environmental reddening gives populations more time to respond to changing conditions (improved environmental tracking), which means that they attain lower densities during periods of starvation and are able to reach their carrying capacities when conditions are favourable. Also resource dynamics should remain relatively independent of environmental conditions, as decreased consumption by one species is compensated by increased consumption by other species in the community (Gonzalez and De Feo, 2007). This means that the mechanism behind the increased consumer variability due to environmental reddening differs from our model, with alternative resources associated with independent environmental variables.

Due to non-linearities in deterministic population dynamics, as well as in the environmental impact, it is not surprising that predictions from simple linear models (e.g., Greenman and Benton, 2005b; Petchey et al., 1997; Ripa and Heino, 1999; Ripa and Ives, 2003) do not necessarily translate to our system and those of others studying the impact of environmental stochasticity in food webs (Gonzalez and De Feo, 2007; Greenman and Benton, 2005a; Vasseur and Fox, 2007). As explained above and in the previous section, the impact of environmental noise does not arise as a simple resonance between the frequencies of the environment and those of population dynamics, but due to its impact on species interactions, via changes in the temporal patterns in stochastic parameters.

\subsection{Adaptive consumer behaviour in noisy environments}

Abrams (1999) suggested that adaptive behaviour on one trophic level generally alters the response of other trophic levels to environmental perturbations. However, little work so far has 
analysed stochastic food web dynamics in association with adaptive behaviour. The only example we know of is given by McCann et al. (2005), who introduced white, uncorrelated noise in resource carrying capacities in quad-trophic food webs with diet switching. When a top predator selected between the top consumers in two distinct food webs, increasingly balanced consumption (i.e., stronger coupling between the food webs by the top predator) promoted stability at the level of the entire system (McCann et al., 2005). Our analysis provides the first example of flexible adaptation in stochastic food webs.

Here we studied how increasing rate of adaptive consumer foraging affects population dynamics, when pink environmental noise (a realistic model for natural environmental fluctuations; Vasseur and Yodzis, 2004) affected either resource growth rates $\left(r_{i}\right)$ or consumer intake rates $\left(I_{i}\right)$. In both cases higher dynamical stability due to increasing environmental amplitude makes the system less sensitive to adaptive behaviour, and increases the relative importance of environmental fluctuations in driving population variability.

When environmental noise is relatively strong, the rate of consumer adaptation appears to have little impact on food web dynamics (Fig. 5). Under fast adaptation the dynamics are indeed very similar, when comparing cases with either stochastic $r_{i}^{\prime}$ s or $I_{i}^{\prime} \mathrm{s}$ (Fig. 6). In both cases uncorrelated variation in the stochastic parameters promotes more asynchronous resource dynamics, which is in turn associated with dampened consumer fluctuations due to reduced overexploitation (e.g., McCann and Rooney, 2009; Rooney et al., 2006). In contrast, slow adaptation resembles random foraging (forages on both resources equally), as the consumer is unable to adapt to the current fitness differences before conditions change again (Fig. 6). This means that, in the case of variable $r_{i}^{\prime}$ s, the competitive suppression of a resource with the lower growth rate is accompanied by the relatively constant consumption pressure, which prevents the resources from recovering until conditions have improved considerably. Under stochastic consumption rates resources tend to fluctuate in synchrony (although there are differences in their temporal densities due to differences in consumption rates), as expected under random foraging in the absence of environmental variation.

\subsection{Model assumptions}

The results presented here are based on the assumption of cyclic consumer-resource dynamics in the absence of environmental variation, produced by an arbitrary choice of parameter values. However, analysing several randomised parameter sets indicates that the results considering the effects of noise amplitude and colour are not sensitive to the specific parameter set used here (Fig. S2A, B). Also, relaxing the assumption of cyclic equilibrium dynamics does not either have a major qualitative influence on the results (Fig. S2C, D). The only clear difference between cyclic and stable dynamics arises when forcing resource growth rates (for all noise colours), which is associated with increased biomass variability with increasing noise amplitude under stable equilibrium dynamics (Fig. S2C). This arises due to increased population variabilities in response to reduced mean densities (as a result of lower mean productivity, Fig. 2A).

While the nature of underlying food web dynamics is of little qualitative importance for the results presented here (Fig. S2), the shape of the species-environment interaction (here assumed to be Gaussian) could potentially play a more important role (e.g., Laakso et al., 2001). It is realistic to assume that, while not necessarily symmetric, species physiological response to varying temperature is unimodal (Dreyer et al., 2001; Englund et al., 2011; Freitas et al., 2007). However, animals can alter their behaviour (foraging, habitat selection, dormancy, etc.) in response to temperature variation (e.g., Magnuson et al., 1979; Post et al., 1999). While considering such, more complicated species-environment interactions are beyond the scope of the current analysis, this is an important challenge for future research.

For simplicity, we have here assumed that species environmental optima are centred at the long-term mean of environmental fluctuations, and that parameters are independently affected by noise. However, while species would have perfectly adapted to certain conditions, changes in these conditions or movement to new habitats are likely result in suboptimal adaptation. In the present context this would mean that the long-term distribution of stochastic parameters would change; they would have lower means and different variances (the variance is maximised at the distance of one standard deviation from the environmental mean; Fowler and Ruokolainen, 2013b). While considering the distance between the environmental mean and species optima would be yet another dimension in the analysis, it would be unlikely that this would have any qualitative impact on the results presented here, assuming that stochastic parameters remain independently distributed.

\subsection{Relevance to empirical systems}

Evidence from experimental systems demonstrates that variation (and long term increase) in ambient temperature can modify the strength of consumer-resource interactions, due to changes in consumer feeding rates (e.g., Barton et al., 2009; Jiang and Morin, 2004; Laws and Joern, 2013; Rall et al., 2010), or food quality (Laws and Joern, 2013). Temperature can be associated with varying feeding rates due to physiological responses (Englund et al., 2011; Sanford, 1999). The net consumer-resource interaction strength can also be affected by varying consumer metabolic rates, which can be associated with periods of predator starvation when the metabolic rate exceeds that of resource ingestion (Rall et al., 2010). Our model predicts that occurrence of such conditions can have important consequences on the stability of prey and predator populations. Feeding rates can also vary due to behavioural responses to temperature. For example, diurnal activity of predatory spiders (and their grasshopper prey) depends on temperature (e.g., Barton et al., 2009; Laws and Joern, 2013), which affects the predator-prey encounter rates. Grasshoppers can also alter their diet preference due to varying predation pressure (Barton et al., 2009; Schmitz and Suttle, 2001). That is, temperature-driven variation in predator activity can lead to cascading effects throughout the food web.

The results presented here show how environmental characteristics, in interaction with the non-linear filtering of environmental fluctuations to biological parameters, impact on population stability in simple food webs. Which ecological process (e.g., resource productivity or consumer resource intake) is affected by environmental conditions further affects the way populations respond to perturbations, and how adaptive behaviour might influence population dynamics. As the model and its predictions make sense in the light of empirical observations, our findings add to the understanding of how biological systems behave under variable environments.

\section{Acknowledgements}

We would like to thank Per Lundberg, Jörgen Ripa, and Veijo Kaitala for helpful comments on our manuscript. We would also like to thank Christopher Klausmeier, Stefan Geritz, and Eva Kisdi for inviting us to contribute to this special issue. LR was funded by the Academy of Finland. 


\section{Appendix A. Supplementary material}

Supplementary data associated with this article can be found in the online version at http://dx.doi.org/10.1016/j.jtbi.2013.08.021.

\section{References}

Abrams, P.A., 1999. The adaptive dynamics of consumer choice. The American Naturalist 153, 83-97.

Abrams, P.A., 2000. The impact of habitat selection on the spatial heterogeneity of resources in varying environments. Ecology 81, 2902-2913.

Abrams, P.A., 2009. When does greater mortality increase population size? The long history and diverse mechanisms underlying the hydra effect. Ecology Letters $12,462-474$

Abrams, P.A., Cressman, R., Krivan, V., 2007. The role of behavioral dynamics in determining the patch distributions of interacting species. The American Naturalist 169, 505-518.

Abrams, P.A., Matsuda, H., 2004. Consequences of behavioral dynamics for the population dynamics of predator-prey systems with switching. Population Ecology 46, 13-25.

Abrams, P.A., Ruokolainen, L., 2011. How does adaptive consumer movement affect population dynamics in consumer-resource metacommunities with homogeneous patches? Journal of the Theoretical Biology 277, 99-110.

Abrams, P.A., Ruokolainen, L., Shuter, B.J., McCann, K.S., 2012. Harvesting creates ecological traps: consequences of invisible mortality risks in predator-prey metacommunities. Ecology 93, 281-293.

Amarasekare, P., 2007. Spatial dynamics of communities with intraguild predation: the role of dispersal strategies. The American Naturalist 170, 819-831.

Amarasekare, P., 2008. Spatial dynamics of foodwebs. Annual Review of Ecology, Evolution, and Systematics 39, 479-500.

Barton, B.T., Beckerman, A.P., Schmitz, O.J., 2009. Climate warming strengthens indirect interactions in an old-field food web. Ecology 90, 2346-2351.

Beckerman, A.P., Petchey, O.L., Warren, P.H., 2006. Foraging biology predicts food web complexity. Proceedings of the National Academy of Sciences 103, 13745-13749.

Butcher, J.C., 2008. Numerical Methods for Ordinary Differential Equations, 2nd ed. Wiley, England, UK.

Dreyer, E., Le Roux, X., Montpied, P., Daudet, F.A., Masson, F., 2001. Temperature response of leaf photosynthetic capacity in seedlings from seven temperate tree species. Tree Physiology 21, 223-232.

Englund, G., Öhlund, G., Hein, C.L., Diehl, S., 2011. Temperature dependence of the functional response. Ecology Letters 14, 914-921.

Fowler, M.S., Ruokolainen, L., 2013a. Confounding environmental colour and distribution shape leads to underestimation of population extinction risk. PloS One 8, e55855.

Fowler, M.S., Ruokolainen, L., 2013b. Colonization, covariance and colour: environmental and ecological drivers of diversity-stability relationships. Journal of the Theoretical Biology 324, 32-41.

Freitas, V., Campos, J., Fonds, M., Van der Veer, H.W., 2007. Potential impact of temperature change on epibenthic predator-bivalve prey interactions in temperate estuaries. Journal of Thermal Biology 32, 328-340.

Gonzalez, A., De Feo, O., 2007. Environmental Variability Modulates the Insurance Effects of Diversity in Non-Equilibrium Communities, The Impact of Environmental Variability on Ecological Systems. Springer, NY, USA, pp. 159-177.

Gouhier, T.C., Guichard, F., Gonzalez, A., 2010. Synchrony and stability of food webs in metacommunities. The American Naturalist 175, E16-E34.

Greenman, J.V., Benton, T.G., 2005a. The frequency spectrum of structured discrete time population models: its properties and their ecological implications. Oikos 110, 369-389.

Greenman, J.V., Benton, T.G., 2005b. The impact of environmental fluctuations on structured discrete time population models: resonance, synchrony and threshold behaviour. Theoretical Population Biology 68, 217-235.

Halley, J.M., 1996. Ecology, evolution and 1f-noise. Trends in Ecology and Evolution $11,33-37$.

Heckmann, L., Drossel, B., Brose, U., Guill, C., 2012. Interactive effects of body-size structure and adaptive foraging on food-web stability. Ecology Letters 15, $243-250$.

Hofbauer, J., Sigmund, K., 1998. Evolutionary Games and Population Dynamics. Cambridge University Press, Cambridge, UK.

Ives, A.R., 2007. Diversity and Stability in Ecological Communities, Theoretical Ecology: Principles and Applications. Oxford University Press, New York, USA, pp. 98-110.

Jiang, L., Morin, P.J., 2004. Temperature-dependent interactions explain unexpected responses to environmental warming in communities of competitors. Journal of Animal Ecology 73, 569-576.

Kaitala, V., Ylikarjula, J., Ranta, E., Lundberg, P., 1997. Population dynamics and the colour of environmental noise. Proceedings of the Royal Society B 264, 943-948.

Kondoh, M., 2003. Foraging adaptation and the relationship between food-web complexity and stability. Science 299, 1388.
Kondoh, M., 2006. Does foraging adaptation create the positive complexity-stability relationship in realistic food-web structure? Journal of the Theoretical Biology 238, 646-651.

Laakso, J., Kaitala, V., Ranta, E., 2001. How does environmental variation translate into biological processes? Oikos 92, 119-122.

Laws, A.N., Joern, A., 2013. Predator-prey interactions in a grassland food chain vary with temperature and food quality. Oikos 122, 977-986.

Lindén, A., Lehikoinen, A., Hokkanen, T., Väisänen, R.A., 2011. Modelling irruptions and population dynamics of the great spotted woodpecker-joint effects of density and cone crops. Oikos 120, 1065-1075.

Ma, B.O., Abrams, P.A., Brassil, C.E., 2003. Dynamic versus instantaneous models of diet choice. The American Naturalist 162, 668-684.

Magnuson, J.J., Crowder, L.B., Medvick, P.A., 1979. Temperature as an ecological resource. American Zoologist 19, 331-343.

Matsuda, H., Hori, M., Abrams, P.A., 1996. Effects of predator-specific defence on biodiversity and community complexity in two-trophic-level communities. Evolutionary Ecology 10, 13-28.

McCann, K., Hastings, A., Huxel, G.R., 1998. Weak trophic interactions and the balance of nature. Nature 395, 794-798.

McCann, K.S., Rasmussen, J.B., Umbanhowar, J., 2005. The dynamics of spatially coupled food webs. Ecology Letters 8, 513-523.

McCann, K.S., Rooney, N., 2009. The more food webs change, the more they stay the same. Philosophical Transactions of the Royal Society B: Biological Sciences $364,1789$.

Petchey, O.L., Gonzalez, A., Wilson, H.B., 1997. Effects on population persistence: the interaction between environmental noise colour, intraspecific competition and space. Proceedings of the Royal Society B 264, 1841.

Post, D.M., Conners, M.E., Goldberg, D.S., 2000. Prey preference by a top predator and the stability of linked food chains. Ecology 81, 8-14.

Post, E., Peterson, R.O., Stenseth, N.C., McLaren, B.E., 1999. Ecosystem consequences of wolf behavioural response to climate. Nature 401, 905-907.

Rall, B.C., Ucic-Pestic, O., Ehnes, R.B., Emmerson, M., Brose, U., 2010. Temperature predator-prey interaction strength and population stability. Global Change Biology 16, 2145-2157.

Rip, J.M.K., McCann, K.S., 2011. Cross-ecosystem differences in stability and the principle of energy flux. Ecology Letters 14, 733-740.

Ripa, J., Heino, M., 1999. Linear analysis solves two puzzles in population dynamics: the route to extinction and extinction in coloured environments. Ecology Letters 2, 219-222.

Ripa, J., Ives, A.R., 2003. Food web dynamics in correlated and autocorrelated environments. Theoretical Population Biology 64, 369-384.

Ripa, J., Lundberg, P., Kaitala, V., 1998. A general theory of environmental noise in ecological food webs. The American Naturalist 151, 256-263.

Rooney, N., McCann, K., Gellner, G., Moore, J.C., 2006. Structural asymmetry and the stability of diverse food webs. Nature 442, 265-269.

Rooney, N., McCann, K.S., Moore, J.C., 2008. A landscape theory for food web architecture. Ecology Letters 11, 867-881.

Rueffler, C., Egas, M., Metz, J.A., 2006. Evolutionary predictions should be based on individual-level traits. The American Naturalist 168, E148-E162.

Ruel, J.J., Ayres, M.P., 1999. Jensen's inequality predicts effects of environmental ariation. Trends in Ecology and Evolution 14, 361-366.

Ruokolainen, L., Abrams, P.A., McCann, K.S., Shuter, B.J., 2011. The roles of spatia heterogeneity and adaptive movement in stabilizing (or destabilizing) simple metacommunities. Journal of the Theoretical Biology 291, 76-87.

Ruokolainen, L., Fowler, M.S., 2008. Community extinction patterns in coloured environments. Proceedings of the Royal Society B 275, 1775-1783.

Ruokolainen, L., Lindén, A., Kaitala, V., Fowler, M.S., 2009a. Ecological and evolutionary dynamics under coloured environmental variation. Trends in Ecology and Evolution 24, 555-563.

Ruokolainen, L., Ranta, E., Kaitala, V., Fowler, M.S., 2009b. Community stability under different correlation structures of species' environmental responses. Journal of the Theoretical Biology 261, 379-387.

Ruokolainen, L., Ripa, J., 2012. The strength of species interactions modifies population responses to environmental variation in competitive communities. Journal of the Theoretical Biology 310, 199-205.

Sanford, E., 1999. Regulation of keystone predation by small changes in ocean temperature. Science 283, 2095-2097.

Schmitz, O.J. Suttle, K.B. 2001. Effects of top predator species on direct and indirect interactions in a food web. Ecology 82, 2072-2081.

Schwager, M., Johst, K., Jeltsch, F., 2006. Does red noise increase or decrease extinction risk? Single extreme events versus series of unfavorable conditions. The American Naturalist 167, 879-888.

Silvola, J., Alm, J., Ahlholm, U., Nykanen, H., Martikainen, P.J., 1996. CO 2 fluxes from peat in boreal mires under varying temperature and moisture conditions. Journal of Ecology, 219-228.

Teng, J., McCann, K.S., 2004. Dynamics of compartmented and reticulate food webs in relation to energetic flows. The American Naturalist 164, 85-100.

Thébault, E., Loreau, M., 2005. Trophic interactions and the relationship between species diversity and ecosystem stability. The American Naturalist 166, 95-114.

Uchida, S., Drossel, B., 2007. Relation between complexity and stability in food webs with adaptive behavior. Journal of the Theoretical Biology 247, 713-722.

Vander Zanden, M.J., Vadeboncoeur, Y., 2002. Fishes as integrators of benthic and pelagic food webs in lakes. Ecology 83, 2152-2161.

Vasseur, D.A., 2007. Populations embedded in trophic communities respond differently to coloured environmental noise. Theoretical Population Biology 72, 186-196. 
Vasseur, D.A., Fox, J.W., 2007. Environmental fluctuations can stabilize food web dynamics by increasing synchrony. Ecology Letters 10, 1066-1074.

Vasseur, D.A., McCann, K.S., 2005. A mechanistic approach for modeling temperaturedependent consumer-resource dynamics. American Naturalist 166, 184-198.
Vasseur, D.A., Yodzis, P., 2004. The color of environmental noise. Ecology 85, 1146-1152. Xu, C., Li, Z., 2003. Population dynamics and the color of environmental noise: a study on a three-species food chain system. Ecological Research 18, 145-154. 